\title{
Endokrinologie und Diabetologie praxisnah
}

Die pädiatrische Endokrinologie und die pädiatrische Diabetologie sind in der neuen Weiterbildungsordnung zu einem gemeinsamen Spezialgebiet zusammengefasst worden. Dem wird durch das Buch der Autoren Hiort, Danne und Wabisch Rechnung getragen. Sie zeigen darin die Grundlagen der Kinderendokrinologie und -diabetologie strukturiert auf. Dazu dienen nicht zuletzt auch die übersichtlich gestalteten Tabellen und schematischen Zeichnungen. Leider bleibt wenig Raum für Fotografien und die wenigen vorhandenen sind oftmals klein und undeutlich.

Alle in dieses Gebiet fallenden Krankheitsbilder werden anschaulich beschrieben. Da die Physiologie und die Diagnostik bis hin zu den speziellen Testverfahren in jeweils gesonderten Kapiteln ausführlich aufgeführt werden, wirken die einzelnen Krankheitsbeschreibungen nicht überfrachtet. Zusätzlich

\section{Das Gehirn verstehen}

Auf 140 Seiten erhält der Leser des vorliegenden Werks eine Vorstellung, wie sein Gehirn funktionieren könnte. Dieter Braus beschreibt, wie neurowissenschaftliche Ergebnisse aus Genetik, Tiermodellen, bildgebenden Verfahren und den Kognitionswissenschaften zu einer Neuorientierung im alltäglichen klinischen Denken führen können. Der Bogen spannt sich von der Basis mit Hirnentwicklung/Neuroanatomie über die zerebrale Plastizität, Genetik, Genderdifferenzen, Ernährung, Schlaf bis hin zu psychiatrischen Erkrankungen.

Wer schon immer ahnte, dass sich eine Tafel Schokolade nicht bei jedem um die Hüften legt, der fühlt sich in diesem Buch bestätigt - auch wenn sich die Konsequenzen daraus nicht ändern lassen. Was Oxytocin, Grehlin, Leptin, Orexin \& Co. in unserem Hypothalamus und Nucleus accumbens anstellen, auch darüber wird der Leser auf den aktuellen Wissensstand gebracht. Es wird klar, wie komplex die zerebralen Abläufe gestaltet sind, abhängig von internen und externen Faktoren, wie vielfältig der Einfluss der Neurohormone ist - und wie wenig wir noch über all dies wissen. Die Erkenntnisse der Neurowissenschaften helfen jedoch, sich endgültig von Mythen des 20. Jahrhun- gehen die Autoren auch auf den Umgang mit chronisch erkrankten Kindern in Familie und Arztpraxis ein.

Insgesamt ist dieses Buch durch seine klare Gliederung trotz der umfangreichen Datenlage als Nachschlagewerk sowohl für den schnellen Überblick als auch zur ausführlichen Informationen geeignet. Durch seine absolute Praxisnähe ist es für den Kinderarzt und auch den Arzt in der Weiterbildung im ambulanten und im stationären Bereich eine gute Hilfe.

Dr. Anke Diederichs

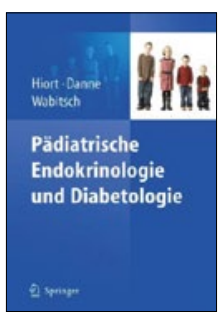

Olaf Hiort et al.

Pädiatrische Endokrinologie und Diabetologie Springer Medizin, Heidelberg 2010 $169,95 €$ ISBN 978-3-642-01911-1

derts zu verabschieden: Autismus entsteht nicht durch die "kaltherzige Mutter" ebenso wenig ADHS durch mangelnde Erziehung. Wie auch bei der Schizophrenie geht man jetzt von "Spektrumerkrankungen“ aus: polygenetisch, multifaktoriell, mit einer weiten klinischen Bandbreite.

Der Autor schafft es, dem Leser den komplexen Wissensstoff anregend und gut verständlich zu vermitteln. Es lohnt sich, das Buch komplett zu lesen und sich nicht nur auf die blau hinterlegte Kapitelzusammenfassungen zu beschränken. Dieses Buch ist jedem an der menschlichen Entwicklung Interessierten zu empfehlen, vor allem aber Kollegen, die sich beruflich mit psychiatrischen/zerebralen Störungen auseinandersetzen. Es hilft, mehr Verständnis für Störungen wie Übergewicht und Schlafstörungen, Angst, Traurigkeit und Sucht zu entwickeln und liefert Argumente, die im therapeutischen Gespräch hilfreich sein können.

Dr. Kirsten Stollhoff

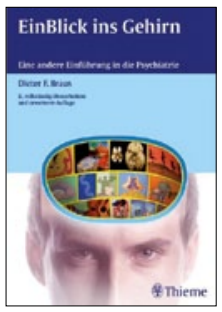

Dieter F. Braus

Ein Blick ins Gehirn

Thieme Verlag, Stuttgart 2011

$39,95 €$

ISBN 3-13-133352-9 